\title{
Transfer of genetic therapy across human populations: molecular targets for increasing patient coverage in repeat expansion diseases
}

\author{
Miguel A Varela ${ }^{\star, 1}$, Helen J Curtis ${ }^{1}$, Andrew GL Douglas ${ }^{1}$, Suzan M Hammond ${ }^{1}$, Aisling J O'Loughlin ${ }^{1}$, \\ Maria J Sobrido ${ }^{2}$, Janine Scholefield ${ }^{1}$ and Matthew JA Wood ${ }^{\star, 1}$
}

\begin{abstract}
Allele-specific gene therapy aims to silence expression of mutant alleles through targeting of disease-linked single-nucleotide polymorphisms (SNPs). However, SNP linkage to disease varies between populations, making such molecular therapies applicable only to a subset of patients. Moreover, not all SNPs have the molecular features necessary for potent gene silencing. Here we provide knowledge to allow the maximisation of patient coverage by building a comprehensive understanding of SNPs ranked according to their predicted suitability toward allele-specific silencing in 14 repeat expansion diseases: amyotrophic lateral sclerosis and frontotemporal dementia, dentatorubral-pallidoluysian atrophy, myotonic dystrophy 1 , myotonic dystrophy 2 , Huntington's disease and several spinocerebellar ataxias. Our systematic analysis of DNA sequence variation shows that most annotated SNPs are not suitable for potent allele-specific silencing across populations because of suboptimal sequence features and low variability ( $>97 \%$ in HD). We suggest maximising patient coverage by selecting SNPs with high heterozygosity across populations, and preferentially targeting SNPs that lead to purine:purine mismatches in wild-type alleles to obtain potent allelespecific silencing. We therefore provide fundamental knowledge on strategies for optimising patient coverage of therapeutics for microsatellite expansion disorders by linking analysis of population genetic variation to the selection of molecular targets. European Journal of Human Genetics (2016) 24, 271-276; doi:10.1038/ejhg.2015.94; published online 20 May 2015
\end{abstract}

\section{INTRODUCTION}

The successful implementation of personalized medicine and, in particular, the development of therapies that silence disease-causing alleles without altering the expression of their wild-type homologues will have a great impact on human health. ${ }^{1,2}$ Most studies on allelespecific silencing are based exclusively on patients of European descent, but given that European populations contain only a subset of human genetic variation, to what degree can such therapeutic methods be readily transferred to patients of different genetic backgrounds? Indeed the heterogeneity of some diseases will make therapies based on a single molecule only applicable to a subset of patients. It could be argued that ultimately additional therapeutic molecules will be developed to permit complete patient coverage. However, a realistic view predicts that the high cost involved in the clinical development of each molecular target will very likely exclude patients with the rarest genotypes.

In this context, acquiring a comprehensive collection of DNA sequence variation, as undertaken by a number of different large-scale genomic projects, ${ }^{3,4,5}$ will provide the information needed to maximise patient coverage using the minimum number of therapeutic molecules targeting the single-nucleotide polymorphisms (SNPs) linked to a disease. Ideally, a list of SNPs would be available for each disease covering all patients (similar to the lists of tag SNPs used for mapping in genome-wide studies), and their corresponding silencing oligomers would be validated as being able to provide potent and allele-specific silencing.
Making this ideal database a reality is not a simple task of ranking SNPs according to their association with the disease. First, when more than one SNP is used to increase the patient coverage, targets must not be completely genetically linked to each other, as the subset of patients included would be entirely overlapping. Second, only some of the sequences containing the SNPs have the molecular features necessary for potent allele-specific gene silencing. ${ }^{6,7,8,9,10}$ Therefore, the future set of guidelines for the gene therapy of these diseases will presumably require iteration from bioinformatics to empirical validation. In this context, taking into account putative targets with their degrees of heterozygosity, haplotype structures across populations and molecular constraints for gene therapy could provide valuable information to facilitate the empirical validation of new allele-specific therapies and predict their performances outside the genotyped sample across different population groups.

In this study we focus on the challenge of developing genetic therapies for microsatellite expansion disorders. Many dominant muscular and neurodegenerative diseases are caused by unstable expansions of microsatellites that induce toxicity at the protein or RNA level. These include the recently discovered hexamer microsatellite causing amyotrophic lateral sclerosis and frontotemporal dementia $^{11,12}$ and other more well-studied microsatellite diseases including myotonic dystrophy 1 and 2, dentatorubral-pallidoluysian atrophy, Huntington's disease, Huntington's disease-like 2 and several spinocerebellar ataxias. ${ }^{13}$

\footnotetext{
${ }^{1}$ Department of Physiology, Anatomy and Genetics, University of Oxford, Oxford, UK; ${ }^{2}$ Fundacion Pública Galega de Medicina Xenómica, Clinical Hospital of Santiago, Travesía da Choupana s/n, Santiago de Compostela, Spain

${ }^{*}$ Correspondence: Dr MA Varela or MJA Wood, Department of Physiology, Anatomy and Genetics, University of Oxford, South Parks Road, Oxford OX1 3QX, UK. Tel: +44 (0) 1865 272167; Fax: +44 (0) 1865 272420; E-mail: miguel.varela@wikisequences.org (MAV) or Tel: +44 (0) 1865 272419; Fax: +44 (0) 1865 272420; E-mail: matthew.wood@dpag.ox.ac.uk (MJA W)

Received 28 April 2014; revised 20 March 2015; accepted 31 March 2015; published online 20 May 2015
} 
Here we combine population genetics analysis and predictors of molecular amenability to gene therapy of each SNP in order to facilitate the optimal targeting of therapeutics in terms of patient coverage and potency. The analysis of population-based sequence variation combined with the validation of molecular targets for potency and patient coverage will likely lead to a future set of guidelines for the treatment of microsatellite expansion diseases.

\section{MATERIALS AND METHODS}

\section{Bioinformatics}

SPSmart (http://spsmart.cesga.es/) was used to retrieve SNPs and their heterozygosity for each gene in populations with African, Asian, and European ancestry, YRI, CHB and CEU respectively: YRI, Yoruba, $n=88$; CEU, Utah residents with Northern and Western European ancestry, $n=87$; CHB, Han Chinese from Beijing, $n=97$ (1000 Genomes Phase I May 2011). A custom VBA macro was written to create the lists of SNPs, assigning each SNP and its features to exons. Haploview was used to build haplotypic blocks (http://www. broadinstitute.org). The limits of haplotype blocks depend on the algorithm employed and marker density. Therefore, blocks must be considered a relative measure of the average linkage disequilibrium for SNPs across populations outside genotyped samples, rather than representing a particular number of haplotypes.

We selected the SNPs discussed in the main text for being exonic, and heterozygous in at least $10 \%$ of the individuals of one of the populations. In Supplementary Material all the SNPs contained on each gene are given with their corresponding heterozygosity in each population, with the only condition that they must be at least $40 \%$ heterozygous in one of the populations. SNPs were classified using a three-colour system in ascending order of their molecular amenability to silencing (red, yellow and green). Note that this classification is based only on the nature of the SNP, and different chemistries can have additional sequence requirements. This is also a relatively conservative classification in that some of the SNPs listed in red may also be amenable to good allele-specificity.

Models of patient coverage were built assuming that patients are heterozygous for the disease-causing allele (this should be true for the vast majority of patients with dominant diseases). The maximum patient coverage for each of the therapeutic molecules targeting a SNP linked to a microsatellite should approximate to $\lim p q *(4) r^{2}$ where $p$ and $q$ are the allele frequencies and $r^{2}$ is the linkage disequilibrium between the targeted SNP and the genetic variant causing the disease. Covering $100 \%$ of the patients targeting one SNP is theoretically possible if linkage to the disease is maximum $\left(r^{2}=1\right)$. In this case all patients would be heterozygous for both the disease-causing allele and the targeted SNP, showing always the same allelic combination. If there is no linkage between the targeted SNP and the mutant microsatellite, patient coverage will depend exclusively on the heterozygosity of the SNP, for example, if $50 \%$ of individuals are heterozygous, the coverage of the treatment should be $\sim 25 \%$ of patients.

\section{Statistics}

Statistical analyses of the data were performed using 'R' (http://www.r-project. org/). All error bars used in this study are SEM. All experiments were performed at least in triplicate and statistical differences between treatments were investigated by $t$-tests.

\section{RESULTS AND DISCUSSION}

SNPs are the most common form of allelic variability. Consequently, they are the main targets for antisense molecules aiming to achieve allele-specific gene silencing. Such antisense molecules are designed to be complementary only to the SNP variant linked to the mutant microsatellite, thereby containing a mismatch with the wild-type allele that ablates mRNA knockdown and reduction of levels of the normal protein. One caveat of this approach lies in the heterogeneity of the disease population, that is, a single linked SNP does not necessarily encompass the majority of patients because the expanded microsatellite can be associated with a range of different haplotypes. In many microsatellite expansion diseases it has been found that the expanded allele has arisen multiple times on different haplotypes. ${ }^{14,15}$ An example of the expansion of a microsatellite occurring in completely different haplotypes is given by SCA36, which was initially described as being exclusive to Japanese patients ${ }^{16}$ but later found to be the cause of the Costa da Morte Ataxia, the most common form of ataxia in the Northwest of Spain ${ }^{17}$ (B, Supplementary Material).

The finding of mutant expansions in multiple haplotypes highlights the need for developing multiple therapeutic molecules targeting different SNPs to cover a greater proportion of patients. A single founder expansion situation would predict identical alleles at all SNP positions close to the mutation, with the strength of linkage decaying smoothly as a function of distance due to the ancestral recombination event. In contrast, expansions on multiple haplotypes would lead to some SNPs being highly associated with the mutant microsatellite with other SNPs that completely lack association. Consequently, the transfer of therapeutic molecules across different human populations will only be possible in some instances owing to their different haplotype structures.

To identify the therapeutic molecules that are most likely to be transferable across different populations, rather than relying exclusively on the linkage information given by a sample of patients comprising only a subset of the genetic heterogeneity of the disease, we suggest also taking into consideration the following features. First, high heterozygosity of the SNPs across populations (eg, on average an SNP with heterozygosity of $50 \%$ across all populations would provide coverage to $25 \%$ of the patients simply by random association with the expanded allele). Second, a relatively high genetic distance from other previously identified SNP targets: on average, SNPs that are adjacent to each other are more likely to be linked compared with SNPs that are located at distant positions within the gene. Two genetically linked SNPs would tend to be present in the same combination of alleles, providing therapy to the same group of genotypes without increasing patient coverage. Choosing distant SNPs would therefore tend to provide a broader combined coverage. Third, SNP nucleotide composition, which can partially predict to what extent allele-specificity would be achievable with a complementary antisense oligonucleotide. For example, SNPs involving purine:purine antisense:target mismatches with the wild-type allele (ie, G:A, G:G, A:A and A:G) will tend to provide the most allele-specific results. ${ }^{6,7}$ Conversely, pyrimidine:pyrimidine, pyrimidine:purine or purine:pyrimidine mismatches are expected to be well accommodated in the antisense moleculemRNA helix and, therefore, display only intermediate levels of discrimination. Of these, the least prone to allele-specific silencing are C:A, U:G, C:U and $\mathrm{U}: \mathrm{U}^{6}$. The G:U mismatch could also result in poor allele-specificity if the antisense molecule contains a modified nucleotide at this site. ${ }^{8}$

In Figures 1 and 2 we show heterozygosity, the amenability of each SNP to be targeted for allele-specific gene therapy and haplotype structure for the HD and SCA7 genes across three populations of African, Asian and European ancestry (Yoruba, CHB and CEU, respectively). The SNPs included are exonic and tailored for maximum silencing efficiency by targeting mRNA using antisense oligonucleotides or siRNAs. Further targets for other genes involved in microsatellite expansion diseases (both with and without including intronic sequences) are described in Supplementary Material.

Optimising antisense molecules for SNPs that contain alleles with a frequency close to $50 \%$ across populations would provide a therapy amenable to transfer between populations. Furthermore, because expansions tend to occur in the most common alleles, the subsets 
of SNPs shown in Figures 1 and 2 and in the Supplementary Material would also tend to include the most useful SNPs in a particular population. As $\mathrm{HD}$ is the most studied microsatellite expansion disease, it provides an example to investigate our ability to predict therapeutic targets with good overall population coverage. For instance, from the 900 or so SNPs of the HTT gene, Warby et al $(2009)^{18}$ and Pfister et al (2009) ${ }^{19}$ genotyped groups of patients and controls and identified one SNP (rs362307) linked to the expanded microsatellite that would be able to provide allele-specific therapy to $\sim 50 \%$ of patients of European ancestry. Pfister et al (2009) then identified and optimised siRNAs for the allele-specific silencing of three SNPs with high heterozygosity that would be able to increase the coverage of patients in their sample to $75 \% .^{18,19}$ All these selected SNPs are present in the group of 25 SNPs given for HD $(2.8 \%$ of all
HTT SNPs) in Figure 1 as being the most heterozygous across different populations in exonic sequences. Likewise, although less work has been devoted to the SCA7 gene, the allele A of the SNP rs3774729 has been reported to be linked to $50 \%$ of patients in South Africa. ${ }^{20}$ This SNP is also in the subset of high heterozygosity SNPs across populations in Figure 2.

It is important to point out that in the case of siRNAs (that target mainly exons, contrary to oligonucleotides) changes in splicing patterns could alter the subset of SNPs that are targetable. This adds an element of uncertainty in the case of siRNAs because different tissues affected by a particular pathology could differ in their splicing patterns. The splicing patterns of all tissues affected by these disorders remain largely unknown but this could constitute a fruitful avenue for further research to refine the subsets of targets.
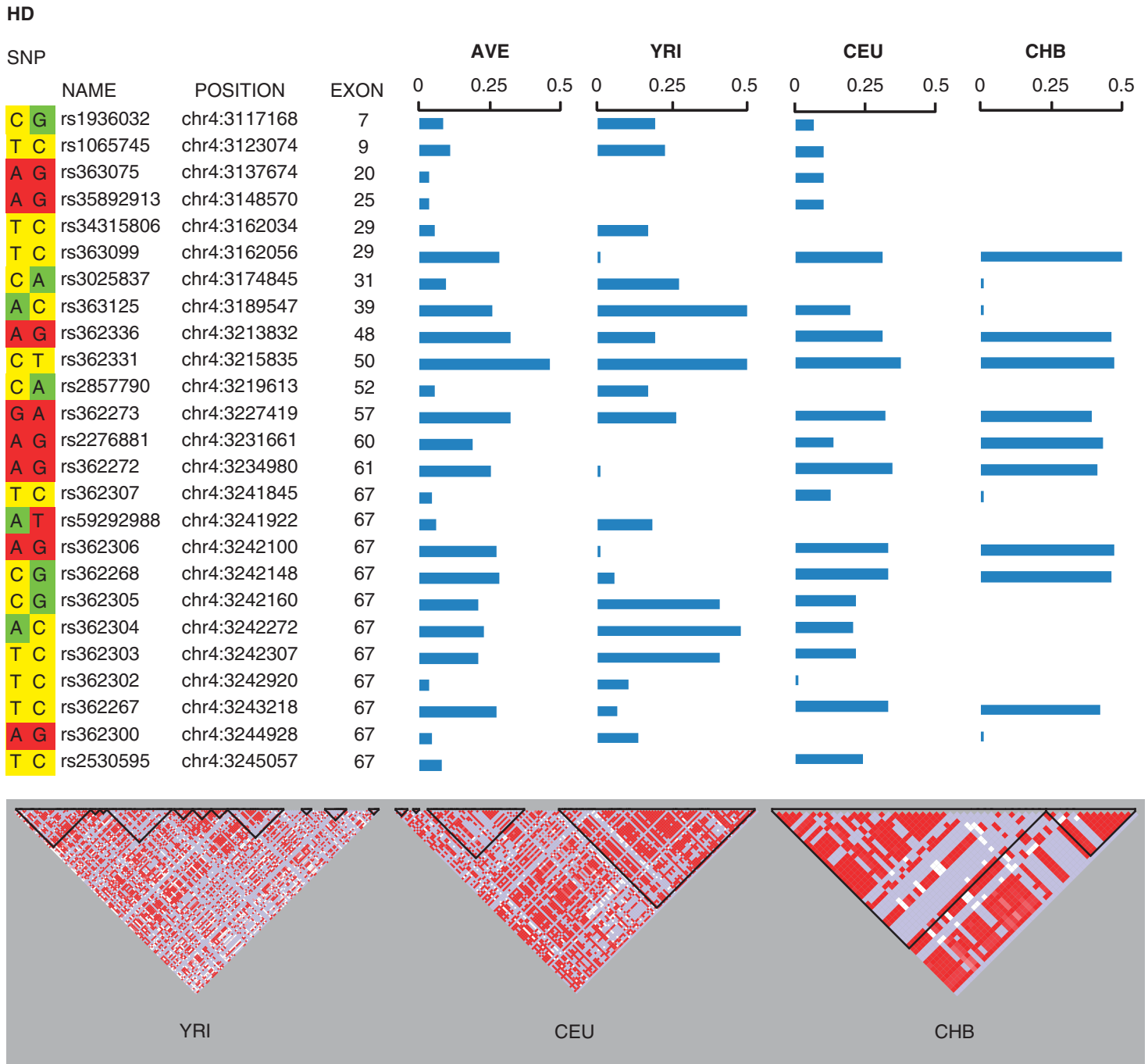

Figure 1 Exonic SNPs in HD selected for having heterozygosity of at least 10\% in one population of Asian (CHB), African (YRI) or European ancestry (CEU). SNPs are classified according to the amenability of each SNP to be targeted for allele-specific gene therapy in ascending order with a three-colour system (red, yellow and green). The first allelic variant is the genomic reference allele and the second the alternative (based on the GRCh37 assembly of the human genome). The colour of each variant predicts the allele-specificity when the variant is contained in the wild type. Thus, it is predicted that targeting a SNP with the wild-type allele highlighted in red will lead to poor allele-specificity (mismatches antisense:target $\mathrm{C}: A, U: G, C: U$ and $U: U$ ) whereas the expression of a wild-type allele in green will tend to remain unchanged (mismatches $G: A, G: G, A: A$ and $A: G$ ), the other mismatches (A:C, G:U, C:C and $U: C$ ) being of intermediate values (wild-type variant in yellow). An overview of linkage disequilibrium by the haplotype blocks is also indicated in the figures below following the standard colour scheme for Haploview in ascending order of linkage: white $=\left(D^{\prime}<1, L O D<2\right)$, pink $=\left(D^{\prime}<1, L O D \geq 2\right), \quad b l u e=\left(D^{\prime}=1, L O D<2\right)$, $r e d=\left(D^{\prime}=1, L O D \geq 2\right)$ (further targets in other genes involved in microsatellite expansion diseases with and without including introns are described in Supplemental Material). 
Finally, both in the case of repeat- and SNP-targeting it is also important to be aware of the unfortunate situation of developing a therapeutic option that is allele-specific but not gene specific, especially if the genes that are targeted are tumour suppressors or have even a more important role than the gene targeted by the treatment. This could be the case of an oligonucleotide treatment claiming to provide a therapy for all HD patients. ${ }^{21}$ The treatment is able to discriminate the wild-type allele of HTT aligning a SNP against the position in the oligonucleotide that provides the highest mismatch discrimination but authors did not report the blast results of the MOE oligonucleotide against other genes. And, in fact, sequences with a single mismatch in positions shown to lack specificity by the authors are also present in 16 other genes (Supplementary Material Table 1S).

\section{Population heterogeneity of allele-specific silencing}

Despite the microsatellite expansions arising in multiple haplotypes, there are cases where a specific haplotype deserves attention. This is applicable where a single ancestral founder can account for the shared haplotype observed in a substantial number of patients (see Supplementary Material for general information about each of these diseases, with an emphasis on population prevalence to help planning the validation of targets). For instance, the highest prevalence of $\mathrm{HD}$ occurs in villages on the coast of Lake Maracaibo (Venezuela) $^{22}$ where there are $>14000$ patients affected (from www.hdfoundation.org).

Apart from differences in prevalence, another population pattern emerges in Figure 3. Patient coverage provided by allele-specific gene therapy depends on population diversity (Figure 3a) and follows a similar pattern to the haplotype coverage of tag SNPs in genome-wide studies. $^{23}$ This is expected by the loss of genetic diversity of human populations with distance from Africa ${ }^{24,25}$ depicted in Figure 3 b by a linear decrease in heterozygosity with land-only geographic distance to Africa of 53 human populations calculated from a 783 microsatellite marker panel. ${ }^{26}$ Therefore, genomes of individuals of African ancestry would provide more targets for allele-specific gene therapy (more SNPs and with higher heterozygosity), and the number of these potential targets would decrease with distance from Africa.

Consequently, in a similar way to the selection of tag SNPs in genetic mapping, a therapeutic molecule targeting one allele linked to a microsatellite would tend to provide coverage to patients in recent
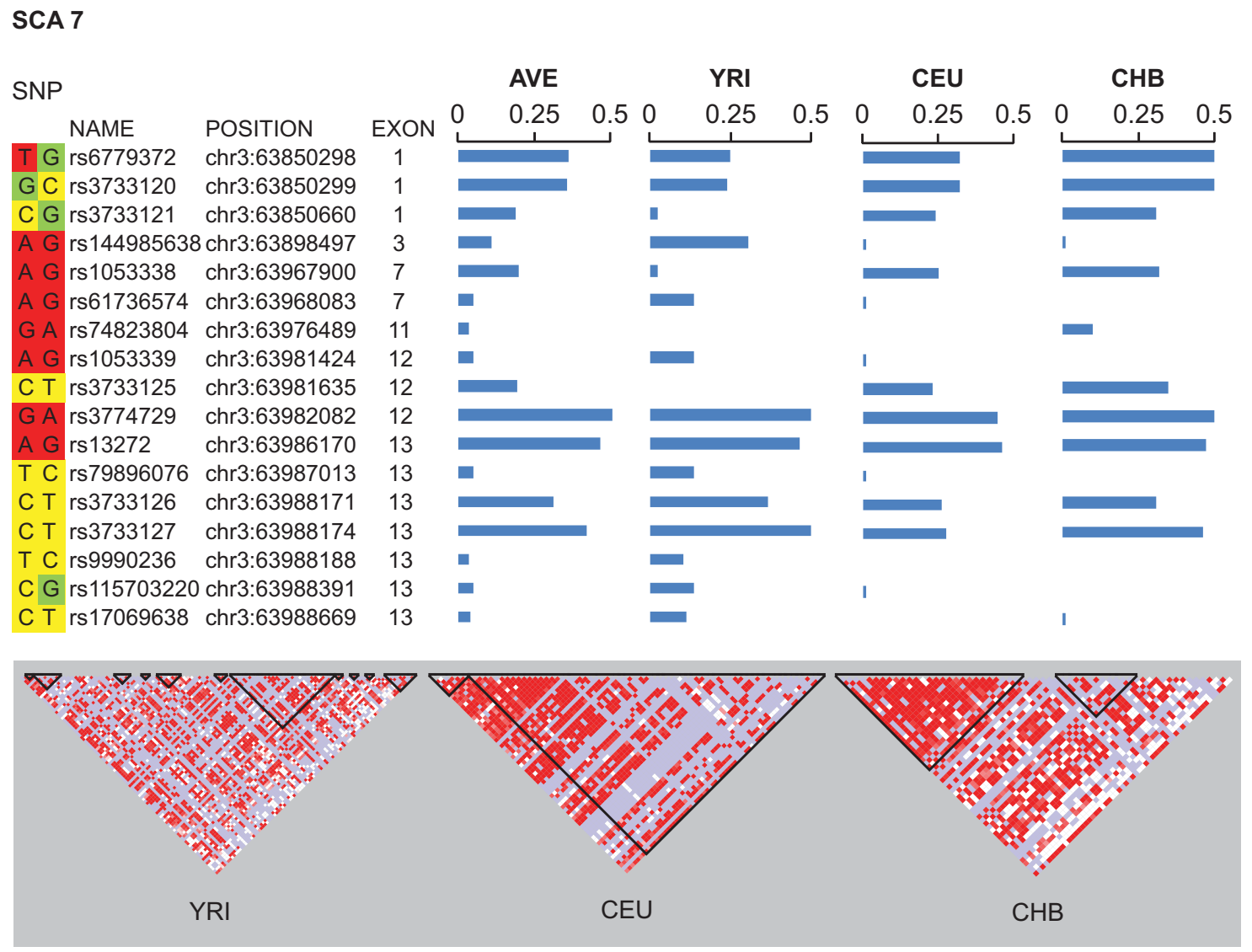

Figure 2 Exonic SNPs in SCA7 selected for having heterozygosity of at least 10\% in one population of Asian (CHB), African (YRI) or European ancestry (CEU). SNPs are classified according to the amenability of each SNP to be targeted for allele-specific gene therapy in ascending order with a three-colour system (red, yellow and green). The first allelic variant is the genomic reference allele and the second the alternative (based on the GRCh37 assembly of the human genome). The colour of each variant predicts the allele-specificity when the variant is contained in the wild type. Thus, it is predicted that targeting a SNP with the wild-type allele highlighted in red will lead to poor allele-specificity (mismatches antisense:target $C: A, U: G, C: U$ and $U: U$ ), whereas the expression of a wild-type allele in green will tend to remain unchanged (mismatches $G: A, G: G, A: A$ and $A: G$ ), the other mismatches (A:C, G:U, C:C and $U: C$ ) being of intermediate values (wild-type variant in yellow). An overview of linkage disequilibrium by the haplotype blocks is also indicated in the figures below following the standard colour scheme for Haploview in ascending order of linkage: white $=\left(D^{\prime}<1, L O D<2\right)$, pink $=\left(D^{\prime}<1, L O D \geq 2\right)$, blue $=\left(D^{\prime}=1, L O D<2\right)$, $r e d=\left(D^{\prime}=1, L O D \geq 2\right)$ (further targets in other genes involved in microsatellite expansion diseases with and without including introns are described in Supplementary Material). 


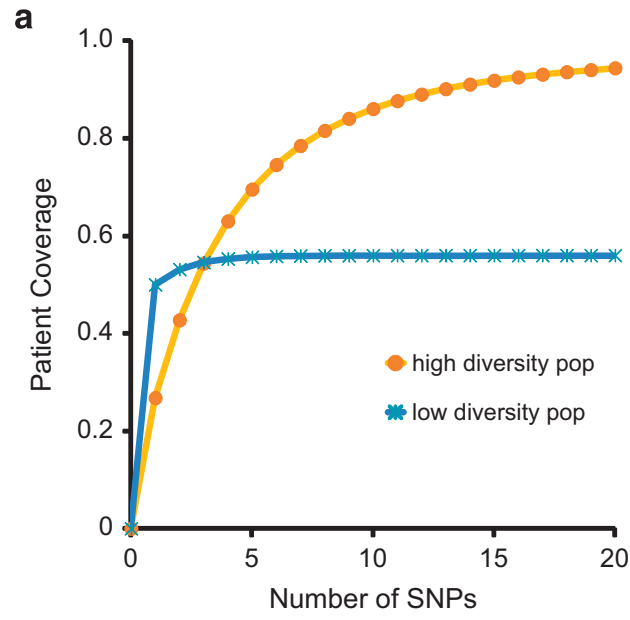

b
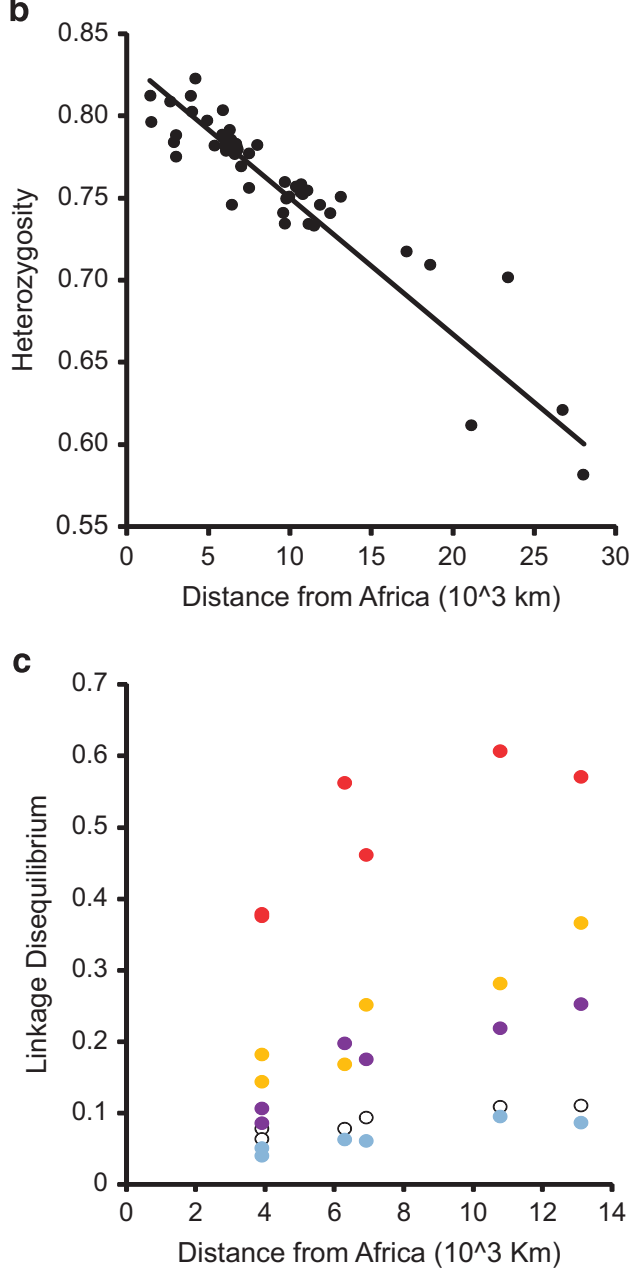

founder effect situations or in low-diversity populations where de novo mutations arise on a similar haplotype background (eg, patients of European ancestry when compared with patients of African descent). However, when we raise the number of therapeutic molecules to increase patient coverage, selecting multiple SNPs from low-diversity populations would likely provide overlapping patient coverage, leaving some patients without any targetable SNPs (Figure 3a). This limit was
Figure 3 Population heterogeneity of allele-specific gene therapy. (a) A therapeutic molecule targeting a single SNP linked to the microsatellite provides coverage to more patients in low-diversity populations but would tend to be linked, saturating patient coverage and leaving more patients without any targetable SNP, contrary to high-diversity populations. Simulations were built using the accumulative coverage provided by targeting several SNPs assuming for the low-diversity population, linkage disequilibrium $r^{2}=0.5$ and for the high-diversity population $r^{2}=0.05$, as described in Materials and Methods. (b) Linear decrease in heterozygosity with increasing land-only geographic distance to Africa of 53 human populations calculated from the 783 microsatellite markers panel of Rosenberg et al. ${ }^{23}$ (c) Average linkage disequilibrium of SNPs for different microsatellite expansion diseases: SCA3 (red), SCA7 (yellow), ALS-FTD (purple), HD (white) and DRPLA (blue), calculated for the following HapMap populations ordered according to distance from Africa: Yoruba, ASW (African ancestry in Southwest USA), Tuscan, CEU (Utah residents with Northern and Western European ancestry), CHB (Han Chinese in Beijing) and JPT (Japanese).

empirically determined by Warby et $a l^{18}$ in their sample of $\mathrm{HD}$ patients of European ancestry, where $6 \%$ of patients were found not to be heterozygous for any exonic SNP. In addition, the lack of targetable SNPs in low-diversity populations is more severe in genes of reduced size, which contain relatively few haplotypes, and aggravated by the low SNP density that tends to occur in sequences flanking long microsatellites. ${ }^{27,28}$

Apart from reduced gene size, there are other factors such as low genetic recombination rates and high levels of natural selection pressure that can contribute to increased average levels of linkage disequilibrium. To exemplify this genomic variability, we show the average linkage disequilibrium of SNPs for several disease-related genes calculated for the HapMap populations ordered according to distance of that population from Africa (Figure 3c). In this figure one can observe the disparity in linkage disequilibrium between the SNPs of different genes (eg, the ATXN3 gene in comparison with HTT) and the tendency of linkage disequilibrium to increase with distance from Africa because of the loss of haplotypes through genetic drift.

\section{CONCLUSIONS}

In this study we described the complexity of transferring allele-specific antisense gene therapy for microsatellite disorders between populations, which leads to the need to develop a number of therapeutic antisense agents to treat a significant percentage of patients. We aimed to facilitate the validation of therapeutic targets by providing predictors of allele-specificity and information to maximise patient coverage while using a minimum number of target SNPs. And we showed that a low-diversity genetic background not only gives a greater chance of developing a single high-coverage antisense molecule but also increases the proportion of patients for which there will be no targetable SNPs. We hope that this work will constitute a fruitful route for future studies that aim to validate new allele-specific treatments for maximum patient coverage across populations.

\section{CONFLICT OF INTEREST}

The authors declare no conflict of interest.

\section{ACKNOWLEDGEMENTS}

This work was supported by Ataxia UK. MAV was funded by Fundacion Pedro Barrie de la Maza. The authors are supported by the Medical Research Council (UK). 
1 Martínez T, Wright N, López-Fraga M, Jiménez Al, Pañeda C. Silencing human genetic diseases with oligonucleotide-based therapies. Hum Genet 2013; 132: 481-493.

2 Seyhan AA: RNAi: a potential new class of therapeutic for human genetic disease Hum Genet 2011; 130: 583-605.

3 Gibbs RA, Belmont JW, Hardenbol P et al: The International HapMap Project. Nature 2003; 426: 789-796.

4 Thorisson GA, Smith AV, Krishnan L, Stein LD: The International HapMap Project Web site. Genome Res 2005; 15: 1592-1593.

5 Ozdemir V, Rosenblatt DS, Warnich L et al: Towards an Ecology of Collective Innovation: Human Variome Project (HVP), Rare Disease Consortium for Autosomal Loci (RaDiCAL) and Data-Enabled Life Sciences Alliance (DELSA). Curr Pharmacogenomics Person Med 2011; 9: 243-251.

$6 \mathrm{Du} \mathrm{Q}$, Thonberg H, Wang J, Wahlestedt C, Liang Z: A systematic analysis of the silencing effects of an active siRNA at all single-nucleotide mismatched target sites. Nucleic Acids Res 2005; 33: 1671-1677.

7 Dykxhoorn DM, Schlehuber LD, London IM, Lieberman J: Determinants of specific RNA interference-mediated silencing of human beta-globin alleles differing by a single nucleotide polymorphism. Proc Natl Acad Sci USA 2006; 103: 5953-5958.

8 You Y, Moreira BG, Behlke MA, Owczarzy R: Design of LNA probes that improve mismatch discrimination. Nucleic Acids Res 2006; 34: e60.

9 Carroll JB, Warby SC, Southwell AL et al: Potent and selective antisense oligonucleotides targeting single-nucleotide polymorphisms in the Huntington disease gene/allele-specific silencing of mutant huntingtin. Mol Ther 2011; 19: 2178-2185.

10 Østergaard ME, Southwell $\mathrm{AL}$, Kordasiewicz $\mathrm{H}$ et al: Rational design of antisense oligonucleotides targeting single nucleotide polymorphisms for potent and allele selective suppression of mutant Huntingtin in the CNS. Nucleic Acids Res 2013; 41: 9634-9650.

11 DeJesus-Hernandez M, Mackenzie IR, Boeve BF et al: Expanded GGGGCC hexanucleotide repeat in noncoding region of C9ORF72 causes chromosome 9p-linked FTD and ALS. Neuron 2011; 72: 245-256.

12 Renton AE, Majounie E, Waite A et al: A hexanucleotide repeat expansion in C90RF72 is the cause of chromosome 9p21-linked ALS-FTD. Neuron 2011; 72: 257-268.

13 Watson LM, Wood MJA RNA: therapy for polyglutamine neurodegenerative diseases. Expert Rev Mol Med 2012; 14: e3.

14 Almqvist EW, Elterman DS, MacLeod PM, Hayden MR: High incidence rate and absent family histories in one quarter of patients newly diagnosed with Huntington disease in British Columbia. Clin Genet 2001; 60: 198-205.

15 Falush D, Almqvist EW, Brinkmann RR, Iwasa Y, Hayden MR: Measurement of mutational flow implies both a high new-mutation rate for Huntington disease and substantial underascertainment of late-onset cases. Am J Hum Genet 2001; 68: 373-385.

16 Kobayashi H, Abe K, Matsuura T et al: Expansion of intronic GGCCTG hexanucleotide repeat in NOP56 causes SCA36, a type of spinocerebellar ataxia accompanied by motor neuron involvement. Am J Hum Genet 2011; 89: 121-130.

17 García-Murias M, Quintáns B, Arias M et al: 'Costa da Morte' ataxia is spinocerebellar ataxia 36: clinical and genetic characterization. Brain 2012; 135: 1423-1435.

18 Warby SC, Montpetit A, Hayden AR et al: CAG expansion in the Huntington disease gene is associated with a specific and targetable predisposing haplogroup. Am J Hum Genet 2009; 84: 351-366.

19 Pfister EL, Kennington L, Straubhaar J et al: Five siRNAs targeting three SNPs may provide therapy for three-quarters of Huntington's disease patients. Curr Biol 2009; 19: 774-778.

20 Greenberg J, Solomon GAE, Vorster AA, Heckmann J, Bryer A: Origin of the SCA7 gene mutation in South Africa: implications for molecular diagnostics. Clin Genet 2006; 70: 415-417.

21 Skotte NH, Southwell AL, Østergaard ME et al: Allele-specific suppression of mutant huntingtin using antisense oligonucleotides: providing a therapeutic option for all Huntington disease patients. PLoS One 2014; 9: e107434.

22 Wexler NS, Lorimer J, Porter J et al: Venezuelan kindreds reveal that genetic and environmental factors modulate Huntington's disease age of onset. Proc Natl Acad Sci USA 2004; 101: 3498-3503.

23 Rosenberg NA, Huang L, Jewett EM, Szpiech ZA, Jankovic I, Boehnke M: Genome-wide association studies in diverse populations. Nat Rev Genet 2010; 11 : 356-366.

24 Prugnolle F, Manica A, Balloux F: Geography predicts neutral genetic diversity of human populations. Curr Biol 2005; 15: R159-R160.

25 Ray N, Currat M, Berthier P, Excoffier L: Recovering the geographic origin of early modern humans by realistic and spatially explicit simulations. Genome Res 2005; 15: 1161-1167.

26 Rosenberg NA, Pritchard JK, Weber JL et al: Genetic structure of human populations. Science 2002; 298: 2381-2385.

27 Varela MA, Sanmiguel R, Gonzalez-Tizon A, Martinez-Lage A: Heterogeneous nature and distribution of interruptions in dinucleotides may indicate the existence of biased substitutions underlying microsatellite evolution. J Mol Evol 2008; 66: 575-580.

28 Varela MA, Amos W: Heterogeneous distribution of SNPs in the human genome: microsatellites as predictors of nucleotide diversity and divergence. Genomics 2010; 95: 151-159.

Supplementary Information accompanies this paper on European Journal of Human Genetics website (http://www.nature.com/ejhg) 